\title{
Arrhythmic and Vascular Complications of Coronavirus Disease 2019 (COVID-19)
}

Editors

DOMENICO G. DELLA ROCCA GIOVANNI B. FORLEO ANDREA NATALE

\section{CARDIAC \\ ELECTROPHYSIOLOGY CLINICS}

www.cardiacEP.theclinics.com

Consulting Editors

RANJAN K. THAKUR

ANDREA NATALE

March 2022 • Volume 14 - Number 1 